\title{
Best Achievable Tracking Performance in Sampled-Data Systems via LTI Controllers
}

\author{
Jie Chen, Fellow, IEEE, Shinji Hara, Fellow, IEEE, Li Qiu, Fellow, IEEE, and Richard H. Middleton, Fellow, IEEE
}

\begin{abstract}
In this paper, we study the problem of tracking a step reference signal using sampled-data control systems. We are interested in the tracking performance, defined as the integral square of the tracking error response between the system's output and the reference input. This performance is deemed the best achievable by a sampled-data controller with a linear time-invariant discrete-time compensator if it is the minimal attainable by all such controllers that stabilize the system. Our primary objective is to investigate the fundamental tracking performance limit in sampled-data systems, and to understand whether and how sampling and hold in a sampled-data system may impose intrinsic barriers to performance. We consider two tracking performance measures, with one defined with respect to the unit step signal, and another with respect to a delayed step signal and averaged over one sampling period. We derive an analytical closed-form expression in each case for the best achievable performance. The results show that a performance loss is generally incurred in a sampled-data system, in comparison to the tracking performance achievable by analog controllers. This loss of performance, as so demonstrated by the expressions, is attributed to the non-minimum phase behaviors as well as the intersample effects generated by samplers and hold devices. Thus, sampled-data controllers do result in an additional performance limit, which is seen as a necessary tradeoff for other advantages offered by this class of controllers.
\end{abstract}

Index Terms-Discretization, frequency-domain lifting, intersample effects, non-minimum phase zeros, performance limit, sampled-data systems, tracking.

\section{INTRODUCTION}

$\mathbf{F}$ OR a given plant, the optimal tracking ability, measured by the minimal tracking error between its output and a reference input to be tracked via a stabilizing controller, depends on the plant, the class of controllers, as well as the reference signal. When the plant and the reference input signal are given, and the controller has been designed, the implementation mode of the controller, i.e. via an analog or a digital controller, will also lead

Manuscript received December 13, 2005; revised December 28, 2006. Current version published December 10, 2008. This work was supported in part by the National Science Foundation (NSF/USA) under Grant ECS-9912533, by the Ministry of Education, Science, Sport and Culture, Japan, under the Grant-in-Aid for COE Research Project of Super Mechano-Systems, by Hong Kong Research Grants Council under the Grant HKUST6212/03E, and by the Natural Science Foundation of China under Grant 60628301. Recommended by Associate Editor D. Henrion.

J. Chen is with the Department of Electrical Engineering, University of California, Riverside, CA 92521-0425 USA (e-mail: jchen@ee.ucr.edu).

S. Hara is with the Department of Information Physics and Computing, The University of Tokyo, Tokyo 113-8656, Japan.

L. Qiu is with the Department of Electronic and Computer Engineering, The Hong Kong University of Science and Technology, Kowloon, Hong Kong.

R. H. Middleton is with The Hamilton Institute, National University of Ireland Maynooth, County Kildare, Ireland.

Digital Object Identifier 10.1109/TAC.2008.2006924 to different tracking performance. In this paper, we consider the tracking performance problem for sampled-data systems, in which the plant operates in continuous time while the controller in discrete time. We consider single-input, single-output (SISO), linear time invariant (LTI) plants. The reference input will be the unit step signal. The tracking performance is defined as the integral square of the error response, measured by the minimal error achievable by all possible sampled-data stabilizing controllers with an LTI compensator. Our main objective is to investigate what may affect the tracking performance in this setting, and whether any limit to this performance may exist, and if any, how and why it arises.

The tracking capability of feedback systems is an important attribute and has been the subject of research for many years. For SISO stable plants, the ability to track step signals with an LTI controller is considered in [21], [24]. It has been shown that the tracking capability is completely determined by the location of non-minimum phase zeros of the plant, whether in continuous time or in discrete time. Recently, these studies have been extended to multi-input multi-output, unstable plants with possible time delays [10], [26], [33], wherein it was found that the tracking performance is determined by the delay times, as well as the location and directional properties of the unstable poles, and the non-minimum phase zeros in the plant, and that the effects of delays, poles and zeros can be completely described via closed-form expressions. A similar conclusion holds with respect to other benchmark signals than the step signal, including sinusoidal and ramp signals [11], [28].

Problems concerning tracking with sampled-data controllers have been widely studied as well; see, e.g., [13]-[15], [18], [19], [36] and the references therein. These problems become considerably more difficult, and closed-form expressions for tracking performance are not yet available; instead, the existing work addresses exclusively numerical design of optimal sampled-data controllers. Among several issues which are unique to sampled-data tracking systems, one important problem is concerned with whether the tracking performance in a sampled-data system may become worse than that of the corresponding analog system. If this is the case, why then is a performance loss incurred in the sampled-data system? Furthermore, what may be the cause contributing to this loss of performance? Would the loss be fundamental of the sampled-data implementation of the controller? If so, can the loss be recovered with sufficiently fast samplers? These issues form the primary objectives of inquiry in the present paper.

The tracking problems being considered in this paper can be posed as sampled-data $\mathcal{H}_{2}$ control problems and tackled using 
existing numerical methods (see, e.g., [13], [15]). It is worth noting that problems of this kind have been studied in a more general setting where linear time-varying (LTV) and periodic controllers are considered [15], [38]. Despite the restriction, the consideration of LTI controllers nevertheless enables us to obtain analytical results that differ considerably from numerical solutions. Specifically, our main results are analytical expressions of the minimal tracking error, whereas the error is defined either as the integral square error in tracking the standard unit step signal originated at the time zero, or as an averaged error in tracking a delayed step reference. These analytical results are crucial for answering the aforementioned questions, which a numerical solution generally fails to address. First, the results show that the time delay and the non-minimum phase zeros of the analog plant continue to impose limits on the sampled-data tracking performance, in exactly the same manner as in using an analog controller. Second, it is well-known that due to the induction of samplers, the ensuing discretization of the plant is likely to generate new non-minimum phase zeros (i.e., zeros outside the unit disc), despite that the original analog plant may itself be minimum phase [2], [3], [6], [17]. The analytical expressions obtained in this paper reveal that such zeros will also have a negative effect on the tracking performance. Third, in order to contain sampling noise and prevent sampling aliases, an analog pre-filter is generally included in a sampled-data system; for the same reason, discretization of this filter may also generate non-minimum phase zeros and hence they too will affect the tracking performance. Finally, our results exhibit further a relationship between the plant's harmonic contents and the tracking performance, showing that the high-frequency harmonics will have a negative effect on the tracking performance as well. In summary, it will be seen that sampling and hold as a whole results in undesirable "byproducts" unfound in analog systems, which contribute to the degradation of the tracking performance.

The notation used throughout this paper is fairly standard. For any complex number $z$, we denote its complex conjugate by $\bar{z}$. For any vector $u$, we denote its transpose by $u^{T}$, and its conjugate transpose by $u^{H}$. The transpose and the conjugate transpose of a matrix $A$ are denoted by $A^{T}$ and $A^{H}$, respectively. We assume that all the vectors and matrices have compatible dimensions, and for simplicity, their dimensions are omitted. Let the open right half plane (RHP) be denoted by $\mathbb{C}_{+}:=\{s: \operatorname{Re}(s)>0\}$, the open left half plane (LHP) by $\mathbb{C}_{-}:=\{s: \operatorname{Re}(s)<0\}$, the imaginary axis by $\mathbb{C}_{0}$, the open unit disc by $\mathbb{D}:=\{z:|z|<1\}$, the exterior of the closed unit disc by $\mathbb{D}^{c}:=\{z:|z|>1\}$, and the unit circle by $\mathbb{T}:=\{z:|z|=1\}$. We will encounter the $\mathcal{L}_{2}$ spaces $\mathcal{L}_{2}\left(\mathbb{C}_{0}\right), \mathcal{L}_{2}(\mathbb{T})$, defined over $\mathbb{C}_{0}, \mathbb{T}$, the $\mathcal{H}_{2}$ spaces together with their orthogonal complements $\mathcal{H}_{2}\left(\mathbb{C}_{+}\right), \mathcal{H}_{2}^{\perp}\left(\mathbb{C}_{+}\right), \mathcal{H}_{2}(\mathbb{D})$, $\mathcal{H}_{2}^{\perp}(\mathbb{D})$, and the $\mathcal{H}_{\infty}$ spaces $\mathcal{H}_{\infty}\left(\mathbb{C}_{+}\right), \mathcal{H}_{\infty}(\mathbb{I})$, and $\mathcal{R} \mathcal{H}_{\infty}(\mathbb{D})$. These spaces correspond to continuous-time $\left(\mathbb{C}_{0}, \mathbb{C}_{+}\right)$and discrete-time $(\mathbb{T}, \mathbb{D})$ frequency responses and transfer functions, and $\mathcal{R H}_{\infty}(\mathbb{I D})$ is the set of all proper stable rational transfer functions in the discrete-time sense; the reader is referred to [13] for the definitions and properties of these spaces.

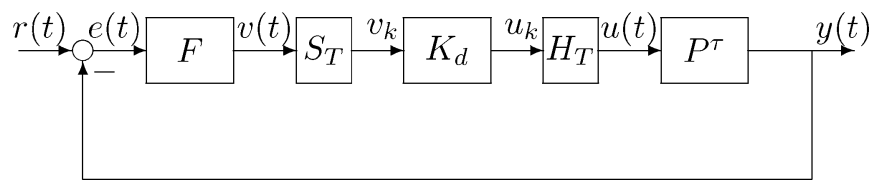

Fig. 1. The sampled-data tracking system.

\section{PRELIMINARIES}

\section{A. Problem Formulation}

We consider the SISO unity feedback system ${ }^{1}$ depicted in Fig. 1, in which $P^{\tau}$ represents the plant model with a possible delay, $F$ a lowpass, anti-aliasing filter, and a sampled-data controller consists of a discrete-time compensator $K_{d}$, followed by a hold device $H_{T}$ and preceded by a sampler $S_{T}$. The signals $r$ and $y$ are the reference input and the system output, respectively. We take $S_{T}$ as an ideal point sampler, and $H_{T}$ a $\mathrm{ZOH}$, which are synchronized and are of the sampling pe$\operatorname{riod} T>0$. Thus, the sampled sequence $\left\{v_{k}\right\}_{k=0}^{\infty}$ is given by $v_{k}:=v(k T), k=0,1, \cdots$, and the $\mathrm{ZOH}$ yields as its output the signal $u(t):=u_{k}$, for $k T \leq t<(k+1) T$.

For a given reference signal $r$, the digital compensator $K_{d}$ is designed such that it stabilizes the analog plant $P^{\tau}$ and the continuous-time output $y$ tracks the continuous-time reference $r$. The signal $e:=y-r$ represents the tracking error response. We take $r$ to be the unit step signal

$$
r(t)=\left\{\begin{array}{ll}
1 & t>0 \\
0 & t<0
\end{array} .\right.
$$

Assume that the system is initially at rest. The problem then is to determine the best tracking performance achievable by all possible digital LTI compensators $K_{d}$ that stabilize the plant. Here we measure the tracking performance by the energy of the tracking error response, denoted as $J_{c}$ and quantified by the integral

$$
J_{c}:=\int_{0}^{\infty}|e(t)|^{2} d t=\int_{0}^{\infty}|y(t)-r(t)|^{2} d t .
$$

With the filter $F$ and the sampling rate $T$ given, the best attainable tracking performance by this class of sampled-data controllers is

$$
J_{s d}^{*}:=\inf _{K_{d}(z)} J_{\text {stabilizes } P^{\tau}(s)} J_{c} .
$$

The minimal $J_{c}$ achievable by an analog controller was found in [10], [24], whose discrete-time counterpart was obtained in [33], both of which admit explicit expressions of the best tracking performance in their respective settings. Our aim in this paper is to derive a corresponding solution for the sampled-data tracking problem, that is, an explicit expression of minimal $J_{c}$ achievable by sampled-data controllers. Alternatively, we will also study a similar performance measure which seeks to average a quadratic

\footnotetext{
${ }^{1}$ Our analysis extends readily to more general two-parameter control systems. For clarity of presentation, and to highlight the fundamental limitation issues, we choose to focus on the unity feedback structure only.
} 
error over a time-shifted reference signal. This alternative performance problem is deferred to Section IV.

Unless otherwise specified, throughout this paper we shall assume

Assumption 1:

1) $P^{\tau}(s)=P(s) e^{-\tau s}$, where $\tau \geq 0$, and $P(s)$ is rational, stable, proper, and $P(0) \neq 0$;

2) $F(s)$ is rational, stable, minimum phase, strictly proper, and $F(0) \neq 0$,

3) $K_{d}(z)$ is rational and proper.

It is worth noting that these assumptions are non-restrictive except the stability assumption on $P(s)$, which can be removed if a two-parameter controller structure is adopted and hence is deemed inessential. ${ }^{2}$ For example, $P(0) \neq 0$ is a standard requirement to ensure that $J_{c}$ be finite. If an anti-aliasing filter $F$ is included in the system, the condition that $F(0) \neq 0$ is also necessary.

For a one-sided signal $g(t)$ with Laplace transform $G(s)$, we denote the $Z$-transform of the sampled sequence $\{g(k T)\}_{k=0}^{\infty}$ by $G_{d}(z)$, and write it as $\mathcal{Z}\{G(s)\}$; that is

$$
G_{d}(z)=\mathcal{Z}\{G(s)\}=\mathcal{Z}\left\{\mathcal{S}_{T}\left\{\mathcal{L}^{-1}\{G(s)\}\right\}\right\}
$$

where $\mathcal{Z}$ is the $\mathcal{Z}$-transform operator, $\mathcal{S}_{T}$ the sampling operator, and $\mathcal{L}^{-1}$ the inverse Laplace transformation. Define

$$
H(s)=\frac{1-e^{-s T}}{s} .
$$

Let $\left(F P^{\tau} H\right)_{d}(z)$ denote the ZOH-equivalent discretization [13], [16] of $F(s) P^{\tau}(s)$, that is

$$
\begin{aligned}
\left(F P^{\tau} H\right)_{d}(z) & =\mathcal{Z}\left\{F(s) P^{\tau}(s) H(s)\right\} \\
& =\left(1-z^{-1}\right) \mathcal{Z}\left[\frac{F(s) P^{\tau}(s)}{s}\right] .
\end{aligned}
$$

It is useful to note that despite the presence of delay, $\left(F P^{\tau} H\right)_{d}(z)$ remains to be a rational function; an explicit construction of such a discretization can be found in, e.g., [16] (pp. 171). Moreover, the stability of $F(s)$ and $P(s)$ guarantees that $\left(F P^{\tau} H\right)_{d}(z)$ is stable. Thus, under Assumption 1, the set of all stabilizing LTI controllers is given by

$$
\begin{aligned}
& \mathcal{K}_{s}:=\left\{K_{d}(z)=Q(z)\left[1-\left(F P^{\tau} H\right)_{d}(z) Q(z)\right]^{-1}:\right. \\
& Q(z)\left.\in \mathcal{R H}_{\infty}(\mathbb{D})\right\} .
\end{aligned}
$$

The optimal tracking performance achievable via an LTI controller can then be determined as

$$
J_{s d}^{*}=\inf _{K_{d} \in \mathcal{K}_{s}} J_{c}=\inf _{Q \in \mathcal{R} \mathcal{H}_{\infty}(\mathbb{D})} J_{c} .
$$

\footnotetext{
${ }^{2}$ Tracking performance with a two-parameter sampled-data controller can be investigated analogously as in this paper, by combining the developments in [1] and [10], which can be shown, as expected, to be unaffected by the plant unstable poles. Thus, in studying fundamental performance limit, the stability assumption does not pose an essential restriction.
}

\section{B. Frequency-Domain Lifting}

We shall tackle the optimal tracking problem from a frequency-domain approach. Let the sampling frequency and the Nyquist frequency be

$$
\omega_{s}=\frac{2 \pi}{T}, \quad \omega_{N}=\frac{\pi}{T}
$$

respectively. We refer to the frequency range $\Omega_{N}=\left[-\omega_{N}, \omega_{N}\right]$ as the baseband. A fundamental fact concerning a sampled signal is that its frequency response consists of infinitely many shifted copies of the original continuous-time frequency response

$$
G_{d}\left(e^{s T}\right)=\frac{1}{T} \sum_{k=-\infty}^{\infty} G\left(s+j k \omega_{s}\right) .
$$

We shall write $G_{k}(s):=G\left(s+j k \omega_{s}\right)$. Consider $G(s):=$ $G^{0}(s) e^{-\tau s}, \tau \geq 0$. By a direct appeal to [7], or a straightforward extension of contour integral argument [25, p. 147] employing the Jordan lemma [22, p. 259], one can show that the above series converges uniformly to $G_{d}\left(e^{s T}\right)$, under the condition that $G^{0}(s)$ is the Laplace transform of the step response of a system whose transfer function is strictly proper and rational. In light of this condition and Assumption 1, we may write

$$
\left(F P^{\tau} H\right)_{d}\left(e^{j \omega T}\right)=\frac{1}{T} \sum_{k=-\infty}^{\infty} H_{k}(j \omega) P_{k}^{\tau}(j \omega) F_{k}(j \omega) .
$$

Let $R(s)$ be the Laplace transform of the reference input $r(t)$ : $R(s)=1 / s$. Then, the output response can be expressed in the frequency domain as

$$
\begin{aligned}
Y(j \omega)=P^{\tau}(j \omega) H(j \omega) S_{d}\left(e^{j \omega T}\right) & K_{d}\left(e^{j \omega T}\right) \\
& \times \frac{1}{T} \sum_{k=-\infty}^{\infty} F_{k}(j \omega) R_{k}(j \omega)
\end{aligned}
$$

where $S_{d}(z):=\left[I+K_{d}(z)\left(F P^{\tau} H\right)_{d}(z)\right]^{-1}$ is the sensitivity function of a discretized system. Employing the parameterization (3), we obtain the error response as

$$
\begin{aligned}
E(j \omega)=R(j \omega)-P^{\tau}(j \omega) H(j \omega) & Q\left(e^{j \omega T}\right) \\
& \times \frac{1}{T} \sum_{k=-\infty}^{\infty} F_{k}(j \omega) R_{k}(\omega)
\end{aligned}
$$

and thus the tracking performance can be expressed in the frequency domain as

$$
J_{c}=\int_{0}^{\infty}|e(t)|^{2} d t=\frac{1}{2 \pi} \int_{-\infty}^{\infty}|E(j \omega)|_{2}^{2} d \omega .
$$

Frequency-domain lifting techniques were developed in, e.g., [1], [8], [18], [37], which have been the subject of numerous studies of sampled-data control problems (see also [38] for an extension to linear, periodically time-varying systems). In what follows we briefly describe this procedure, while referring to [1], [8] for much of the technical detail. Let $E(j \omega) \in \mathcal{L}_{2}\left(\mathbb{C}_{0}\right)$. Define, on the interval $\Omega_{N}$, the sequence of functions

$$
E_{k}(j \omega)=E\left(j \omega+j k \omega_{s}\right), \quad k=0,1, \cdots .
$$


We may arrange the sequence $\left\{E_{k}(j \omega)\right\}_{k=-\infty}^{\infty}$ as an infinitedimensional vector $\mathcal{E}(j \omega)$, that is

$$
\mathcal{E}(j \omega):=\left[\begin{array}{lllll}
\cdots & E_{1}(j \omega) & E_{0}(j \omega) & E_{-1}(j \omega) & \cdots
\end{array}\right]^{T} .
$$

This operation can be described by the linear lifting operator $\mathcal{T}: \mathcal{L}_{2}\left(\mathbb{C}_{0}\right) \rightarrow \mathcal{L}_{2}\left(\Omega_{N}\right)$, such that $\mathcal{T} E(j \omega)=\mathcal{E}(j \omega)$, where $\mathcal{L}_{2}\left(\Omega_{N}\right)$ is the Hilbert space with the inner product

$$
\langle\mathcal{X}, \mathcal{Y}\rangle_{\mathcal{L}_{2}\left(\Omega_{N}\right)}:=\frac{1}{2 \pi} \int_{\Omega_{N}}\left(\sum_{k=-\infty}^{\infty} \bar{X}_{k}(j \omega) Y_{k}(j \omega)\right) d \omega
$$

and the norm

$$
\|\mathcal{Y}\|_{\mathcal{L}_{2}\left(\Omega_{N}\right)}=\left(\frac{1}{2 \pi} \int_{\Omega_{N}}\left(\sum_{k=-\infty}^{\infty}\left|Y_{k}(j \omega)\right|^{2}\right) d \omega\right)^{1 / 2} .
$$

It is known [1], [8] that

$$
\begin{aligned}
\langle X, Y\rangle & =\langle\mathcal{X}, \mathcal{Y}\rangle_{\mathcal{L}_{2}\left(\Omega_{N}\right)} \\
\|E\|_{2} & =\|\mathcal{E}\|_{\mathcal{L}_{2}\left(\Omega_{N}\right)} .
\end{aligned}
$$

Consequently, the tracking performance $J_{c}$ can be quantified using the lifted version of $E(j \omega)$; specifically

$$
J_{c}=\|\mathcal{E}\|_{\mathcal{L}_{2}\left(\Omega_{N}\right)}^{2} .
$$

Denote $\mathcal{P}^{\tau} \mathcal{H}(j \omega)$

$$
\begin{array}{r}
\mathcal{P}^{\tau} \mathcal{H}(j \omega)=\left[\begin{array}{lll}
\cdots & P_{1}^{\tau}(j \omega) H_{1}(j \omega) & P_{0}^{\tau}(j \omega) H_{0}(j \omega) \\
& P_{-1}^{\tau}(j \omega) H_{-1}(j \omega) & \cdots
\end{array}\right]^{T} .
\end{array}
$$

It follows that

$$
\mathcal{E}(j \omega)=\left[\mathcal{I}-\frac{1}{T} \mathcal{P}^{\tau} \mathcal{H}(j \omega) Q\left(e^{j \omega T}\right) \mathcal{F}^{T}(j \omega)\right] \mathcal{R}(j \omega) .
$$

Here, in the last expression, $\mathcal{I}$ is the unit operator on the lifted signal space $\mathcal{L}_{2}\left(\Omega_{N}\right)$, such that $\mathcal{I E}(j \omega)=\mathcal{E}(j \omega)$. The composite operator $\mathcal{P}^{\tau} \mathcal{H} Q \mathcal{F}^{T}$ is known as an $F R$-operator [1], [18]. In light of (7), the best achievable tracking performance can then be found by solving the optimal model-matching problem

$$
J_{s d}^{*}=\inf _{Q \in \mathcal{R} \mathcal{H}_{\infty}(\mathbb{D})}\left\|\left[\mathcal{I}-\frac{1}{T} \mathcal{P}^{\tau} \mathcal{H} Q \mathcal{F}^{T}\right] \mathcal{R}\right\|_{\mathcal{L}_{2}\left(\Omega_{N}\right)}^{2} .
$$

Our goal is obtain an analytical solution to this problem.

\section{Tracking Performance With Unit SteP}

Our main result is given in the following theorem, which is a closed-form, analytical expression of the best tracking performance achievable by a sampled-data control scheme, in which the discrete-time controller $K_{d}$ is assumed to be LTI. We shall need the allpass factorization of $P(s)$, given as

$$
P(s)=L(s) P^{(m)}(s)
$$

where $P^{(m)}(s)$ represents the minimum phase part of $P(s)$, and $L(s)$ is an allpass factor, such that

$$
L(s):=\prod_{i=1}^{m_{c}} \frac{\bar{z}_{i}\left(z_{i}-s\right)}{z_{i}\left(\bar{z}_{i}+s\right)}
$$

with $z_{i} \in \mathbb{C}_{+}, i=1, \cdots, m_{c}$, being the non-minimum phase zeros of $P(s)$.

Theorem 1: Let the reference input $r(t)$ be the step signal given by (1). Then under Assumption 1

$$
J_{s d}^{*}=J_{p}+J_{f}
$$

where

$$
\begin{aligned}
J_{p} & :=\tau+\sum_{i=1}^{m_{c}} \frac{2}{z_{i}}+T \sum_{i=1}^{m_{p}} \frac{\sigma_{i}+1}{\sigma_{i}-1}+J_{h}(T) \\
J_{f} & :=T \sum_{i=1}^{m_{f}} \frac{\lambda_{i}+1}{\lambda_{i}-1}
\end{aligned}
$$

and $J_{h}(T) \geq 0$ is defined as

$$
\begin{aligned}
J_{h}(T) & :=\frac{T^{2}}{2 \pi} \int_{0}^{\omega_{N}} \frac{1}{1-\cos \omega T} \\
& \times \log \left\{\frac{T^{2} \sum_{k=-\infty}^{\infty} \frac{\left|P_{k}^{(m)}(j \omega)\right|^{2}}{\left(\omega+k \omega_{s}\right)^{2}}}{2(1-\cos \omega T)\left|\sum_{k=-\infty}^{\infty} \frac{P_{k}^{(m)}(j \omega)}{\left(\omega+k \omega_{s}\right)^{2}}\right|^{2}}\right\} d \omega
\end{aligned}
$$

with $z_{i} \in \mathbb{C}_{+}, i=1, \cdots, m_{c}$, being the non-minimum phase zeros of $P(s), \sigma_{i} \in \mathbb{D}^{c}, i=1, \cdots, m_{p}$, the non-minimum phase zeros of $z\left(P^{(m)} R H\right)_{d}(z)=z \mathcal{Z}\left\{P^{(m)}(s) R(s) H(s)\right\}$, $\lambda_{i} \in \mathbb{D}^{c}, i=1, \cdots, m_{f}$ the non-minimum phase zeros of $(F H)_{d}(z)=\mathcal{Z}\{F(s) H(s)\}$, with all non-minimum phase zeros of the discrete-time transfer functions counting the zeros at infinity.

Proof: See Appendix A.

We remark that the singular integral in $J_{h}(T)$ converges, a technicality that we choose to omit herein but will become selfevident in the proof of Theorem 1; alternatively, it is straightforward, though somewhat tedious, to show that the integrand has a removable singularity at $\omega=0$.

The quantity $J_{p}$ in (10) represents the performance limit inherent of the plant, in which the first two terms, i.e.,

$$
\tau+\sum_{i=1}^{m_{c}} \frac{2}{z_{i}}
$$

arise due to the time delay and the non-minimum phase zeros of the continuous-time plant. This term coincides with the minimal tracking error attainable by the optimal analog controller. Thus, Theorem 1 shows that the influence on the tracking performance by the nonminimum phase zeros and the time delay remain in complete existence, in exactly the same way, when the optimal analog controller is replaced by a sampled-data controller. The theorem makes it clear that with a discrete-time LTI controller, sampled-data control will generally worsen the tracking performance, due to the presence of the additional terms, which are all non-negative. Since the sampled-data controllers constitute a special class of LTV controllers, this result reinforces the previous finding in [27], that the optimal tracking performance achievable via an LTI or an LTV analog controller coincides. On the other hand, it is also known [15] that with the sampled-data scheme, $J_{s d}^{*}$ can be further improved if the discrete-time LTI 
controller is allowed to be time-varying, which may serve as an even more fundamental measure of tracking performance.

The third and fourth terms in $J_{p}$ are seen as the undesirable "byproduct" of the sampled-data tracking scheme, capturing the negative effect of the sampling and hold operations on the tracking performance. The third term is attributed to the non-minimum phase zeros of an auxiliary discretized system, with a ZOH-equivalent discretization of $P^{(m)}(s) R(s)$; a similar effect arises with the anti-aliasing filter, represented by $J_{f}$. Together with the third, the fourth term in $J_{p}$ manifests in a more explicit fashion the effect of intersample behavior, an important aspect of sampled systems, displaying an explicit dependence of the tracking performance on the high-frequency harmonics of $P^{(m)}(s)$. It is useful to note that this effect is independent of the anti-aliasing filter $F$.

It is well-known that sampled systems are prone to non-minimum phase zeros. In particular, it is known [2], [3], [17] that for a continuous-time system with pole-zero excess greater than two, the sampled system with a ZOH will always result in zeros outside the unit disc, provided that the sampling period is sufficiently small. It is also known that a continuous-time system with pole-zero excess greater or equal to one results in a discrete-time system with a zero at the point infinity, and hence the sampled system becomes non-minimum phase. Theorem 1 shows that such discrete-time non-minimum phase zeros, known as the sampling zeros, as well as the high-frequency harmonics, are the very reason why it is difficult for a sampled-data system to retain the tracking performance achievable by an analog controller.

In summary, it is clear that sampled-data controllers will in general lead to a degradation in the tracking performance. A plausible question is whether with a fast sampling rate, this degradation can be made small. Toward this end, we first note that the third term in $J_{p}$ will diminish, and so will $J_{f}$. Indeed, according to [2] (see also [3], [6], [17]), for sufficiently small $T>0$, the transfer function $\left(P^{(m)} R H\right)_{d}(z)$ will contain zeros mapped from the zeros of the minimum phase transfer functions $P^{(m)}(s) R(s)$, approaching the point $z=1$ from the interior of the unit disc. The remaining zeros of $\left(P^{(m)} R H\right)_{d}(z)$ will approach those of the so-called Euler polynomial. As such, for sufficiently small $T>0$, the second term in $J_{p}$ is only determined by the zeros of the Euler polynomial, which are always real negative. Consequently, we claim that for sufficiently small $T>0$

$$
T \sum_{i=1}^{m_{p}} \frac{\sigma_{i}+1}{\sigma_{i}-1}=O(T), \quad T \sum_{i=1}^{m_{f}} \frac{\lambda_{i}+1}{\lambda_{i}-1}=O(T) .
$$

More generally, it turns out that the optimal performance $J_{s d}^{*}$ as a whole will decrease at a linear rate to the performance achievable by the optimal analog controller. We state this result in the following corollary. The proof is given in Appendix B.

Theorem 2: Under the assumptions in Theorem 1, as $T \rightarrow 0$

$$
J_{s d}^{*}=\tau+\sum_{i=1}^{m_{c}} \frac{2}{z_{i}}+O(T) .
$$

It is then clear that the effects of sampling and hold will become negligible for sufficiently small $T>0$; in other words, the performance loss due to the use of sampled-data controllers can be recovered in the limit, with an arbitrarily fast sampler.

\section{Average Tracking Performance}

An alternative, complementary measure that can be used to quantify the tracking performance is the $\mathcal{H}_{2}$ performance criterion that seeks to average the quadratic error in (2), over a time-shifted reference signal. In this formulation, we consider the tracking error defined by

$$
J(\theta):=\int_{0}^{\infty}|y(t)-r(t-\theta)|^{2} d t, \quad 0 \leq \theta \leq T .
$$

Note that $J(0)=J_{c}$. The averaged tracking performance is defined as

$$
J^{a}:=\frac{1}{T} \int_{0}^{T} J(\theta) d \theta
$$

and the optimal average performance becomes

$$
J_{s d}^{a}:=\inf _{K_{d}(z)} J_{\text {stabilizes } P(s)} J^{a} .
$$

Averaged $\mathcal{H}_{2}$ performance measures in the spirit of (14) were advocated in [5], [12], [20] for sampled-data systems, and studied in [38] for more general periodic LTV systems. Our following result, a counterpart to Theorem 1, gives an analytical expression as well for the optimal average tracking performance.

Theorem 1: Let the reference input $r(t)$ be the step signal given by (1). Let also $\left(A_{f}, B_{f}, C_{f}\right)$ be a minimal realization of $F(s)$. Define

$$
\begin{aligned}
A_{f d} & :=e^{A_{f} T} \\
B_{f d} & :=\int_{0}^{T} e^{A_{f} t} B_{f} d t \\
B_{f}(t) & :=\int_{0}^{t} e^{A_{f} \tau} B_{f} d \tau \\
B_{f a} & :=\frac{1}{T} \int_{0}^{T} B_{f}(t) d t \\
\Sigma_{f} & :=\frac{1}{T} \int_{0}^{T} B_{f}(t) B_{f}^{T}(t) d t \\
\Lambda_{f} & :=\left(\Sigma_{f}-B_{f a} B_{f a}^{T}\right)^{1 / 2} \\
F_{a 1}(z) & :=(F H)_{d}(z)+(z-1) C_{f}\left(z I-A_{f d}\right)^{-1} B_{f a} \\
F_{a 2}(z) & :=(z-1) C_{f}\left(z I-A_{f d}\right)^{-1} \Lambda_{f} .
\end{aligned}
$$

Then under Assumption 1

$$
J_{s d}^{a}=\frac{T}{2}+J_{p}+J_{f}^{a}
$$


where $J_{p}$ is given as in Theorem 1

$$
\begin{aligned}
J_{f}^{a} & :=T \sum_{i=1}^{m_{a}} \frac{\gamma_{i}+1}{\gamma_{i}-1}+J_{h}^{a}(T) \\
J_{h}^{a}(T) & :=\frac{T^{2}}{2 \pi} \int_{0}^{\omega_{N}} \frac{1}{1-\cos \omega T} \log \left(1+\frac{\left\|F_{a 2}\left(e^{j \omega T}\right)\right\|^{2}}{\left|F_{a 1}\left(e^{j \omega T}\right)\right|^{2}}\right) d \omega
\end{aligned}
$$

and $\gamma_{i} \in \mathbb{D}^{c}, i=1, \cdots, m_{a}$, are the non-minimum phase zeros of $F_{a 1}(z)$ counting the zeros at infinity.

Proof: See Appendix C.

It is clear that the essential difference between $J_{s d}^{*}$ and $J_{s d}^{a}$ results from the use of the anti-aliasing filter $F$. On the other hand, the performance limit due to the plant, i.e., the term $J_{p}$ is invariant of the measures and thus appears more fundamental to the tracking performance. Theorem 1 and Theorem 2 , therefore, reinforce each other, demonstrating the fundamental role of $J_{p}$ in a sampled-data tracking system regardless of the performance measures adopted.

Analogously, the following asymptotic property of $J_{s d}^{a}$ can be established. The proof is similar to that for Theorem 2 and thus omitted.

Theorem 4: Under the assumptions in Theorem 1, as $T \rightarrow 0$

$$
J_{s d}^{a}=\tau+\sum_{i=1}^{m_{c}} \frac{2}{z_{i}}+O(T)
$$

We note that the convergence of $J_{s d}^{a}$ was previously established in [34]. However, Theorem 4, much like Theorem 2, presents a stronger characterization of this convergence, establishing a linear convergence rate.

\section{CONCLUSION}

In this paper we have derived explicit expressions for the optimal performance in tracking a step reference signal via sampled-data controllers. Our results demonstrate that the best tracking performance achievable by LTI controllers is negatively affected by 1) the time delay and non-minimum phase zeros of the continuous-time plant; 2) the non-minimum phase zeros resulted from discretization; and 3) the effects due to sampling and hold operations, attributed to both sampling zeros and the plant high-frequency harmonics. While the first source inherits completely and exactly from the analog tracking system, the rest results from the use of a sampled-data controller. Nevertheless, it is also shown that with an arbitrarily fast sampler, this tracking performance does approach asymptotically that achievable by an analog controller, hence recovering in full the performance loss incurred by the sampling and hold operations.

The present work can be extended in a straightforward manner to multi-input multi-output systems, using a combination of the techniques developed herein and in [9], [10]. It can also be readily generalized to systems with unstable plants. In the latter vein, two-parameter controllers can be used to achieve the optimal performance, which as shown in [9], [10] for analog systems and in [29], [33] for discrete-time systems, are exempted from the effect of plant unstable poles. Indeed, it can be shown, by combining the development in [1] (where a two-parameter sampled-data control structure was considered) and the techniques in [10] and the present paper, that this remains true for sampled-data systems, and that in fact, despite the presence of plant unstable poles, the optimal tracking performance achievable by a two-parameter sampled-data controller will coincide with that in the present setting, i.e., a stable plant controlled by a one-parameter sampled-data controller. As such, our study of stable plants suffices to expose the fundamental limit to the achievable tracking performance, which is solely determined by the non-minimum phase behavior of the plant and the intersample effect of a sampled system.

\section{APPENDIX A \\ PROOF OF THEOREM 1}

Proof: The proof of Theorem 1 requires a lengthy derivation. Our first step is to separate the non-minimum phase zeros of $P(s)$. For this purpose, we invoke the allpass factorization (9). Introducing the diagonal operators $\mathcal{D}(j \omega)$, $\mathcal{L}(j \omega): \mathcal{L}_{2}\left(\Omega_{N}\right) \rightarrow \mathcal{L}_{2}\left(\Omega_{N}\right)$, so that

$$
\begin{aligned}
\mathcal{L}(j \omega) & :=\operatorname{diag}\left(\cdots, L_{1}(j \omega), L_{0}(j \omega), L_{-1}(j \omega), \cdots\right) \\
\mathcal{D}(j \omega) & :=\operatorname{diag}\left(\cdots, e^{-j \tau\left(\omega+\omega_{s}\right)}, e^{-j \tau \omega}, e^{-j \tau\left(\omega-\omega_{s}\right)}, \cdots\right) .
\end{aligned}
$$

It follows that $\mathcal{P}^{\tau} \mathcal{H}(j \omega)=\mathcal{D}(j \omega) \mathcal{L}(j \omega) \mathcal{P}^{(m)} \mathcal{H}(j \omega)$, where:

$$
\begin{array}{r}
\mathcal{P}^{(m)} \mathcal{H}(j \omega):=\left[\begin{array}{lll}
\cdots & P_{1}^{(m)}(j \omega) H_{1}(j \omega) & P_{0}^{(m)}(j \omega) H_{0}(j \omega) \\
& P_{-1}^{(m)}(j \omega) H_{-1}(j \omega) & \cdots
\end{array}\right]^{T} .
\end{array}
$$

We claim that

$$
J_{s d}^{*}=\tau+\sum_{i=1}^{m_{c}} \frac{2}{z_{i}}+J_{m}^{*}
$$

with

$$
J_{m}^{*}:=\inf _{Q \in \mathcal{R} \mathcal{H}_{\infty}(\mathbb{D})}\left\|\left[I-\frac{1}{T} \mathcal{P}^{(m)} \mathcal{H} Q \mathcal{F}^{T}\right] \mathcal{R}\right\|_{\mathcal{L}_{2}\left(\Omega_{N}\right)}^{2} .
$$

Indeed, since $\left(e^{\tau s}-1\right) R(s) \in \mathcal{H}_{2}^{\perp}\left(\mathbb{C}_{+}\right)$and $\left(L^{-1}(s)-\right.$ 1) $R(s) \in \mathcal{H}_{2}^{\perp}\left(\mathbb{C}_{+}\right)$, it follows analogously as in [10] that:

$$
\begin{aligned}
J_{s d}^{*} & =\left\|\left(\mathcal{D}^{-1}-\mathcal{I}\right) \mathcal{R}\right\|_{\mathcal{L}_{2}\left(\Omega_{N}\right)}^{2}+\left\|\left[\mathcal{L}^{-1}-\mathcal{I}\right] \mathcal{R}\right\|_{\mathcal{L}_{2}\left(\Omega_{N}\right)}^{2}+J_{m}^{*} \\
& =\left\|\left(e^{j \tau \omega}-1\right) R\right\|_{2}^{2}+\left\|\left[L^{-1}-1\right] R\right\|_{2}^{2}+J_{m}^{*} \\
& =\tau+\sum_{i=1}^{m_{c}} \frac{2}{z_{i}}+J_{m}^{*} .
\end{aligned}
$$

We next evaluate $J_{m}^{*}$. Define

$$
\begin{aligned}
\Phi_{d}\left(e^{j \omega T}\right) & :=\frac{1}{T} \sum_{k=-\infty}^{\infty}\left|P_{k}^{(m)}(j \omega) H_{k}(j \omega)\right|^{2} \\
& =\frac{1}{T} \mathcal{P}^{(m)} \mathcal{H}^{H}(j \omega) \mathcal{P}^{(m)} \mathcal{H}(j \omega) .
\end{aligned}
$$


It is possible to carry out a spectral factorization [13], [35] for $\Phi_{d}\left(e^{j \omega T}\right)$, such that

$$
\Phi_{d}\left(e^{j \omega T}\right)=\Theta_{d}\left(e^{-j \omega T}\right) \Theta_{d}\left(e^{j \omega T}\right) .
$$

We note that $\Phi_{d}\left(e^{j \omega T}\right)$ can be constructed explicitly as a rational function [8], which ensures that $\Theta_{d}(z) \in \mathcal{R} \mathcal{H}_{\infty}(\mathbb{I D})$, and $\Theta_{d}^{-1}(z) \in \mathcal{R} \mathcal{H}_{\infty}(\mathbb{D})$. Note that

$$
\left|\Theta_{d}(1)\right|^{2}=\frac{1}{T} \sum_{k=-\infty}^{\infty}\left|P_{k}(0) H_{k}(0)\right|^{2}
$$

Since

$$
H_{k}(0)= \begin{cases}0 & k \neq 0 \\ T & k=0\end{cases}
$$

we have $\left|\Theta_{d}(1)\right|^{2}=T \cdot|P(0)|^{2}$. Without loss of generality, we may take

$$
\Theta_{d}(1)=\sqrt{T} P(0)
$$

Define the infinite dimensional vector

$$
\mathcal{M}_{i}(j \omega)=\frac{1}{\sqrt{T}} \mathcal{P}^{(m)} \mathcal{H}(j \omega) \Theta_{d}^{-1}\left(e^{j \omega T}\right)
$$

and the operator

$$
\mathcal{W}(j \omega):=\left[\begin{array}{c}
\mathcal{M}_{i}^{H}(j \omega) \\
\mathcal{I}-\mathcal{M}_{i}(j \omega) \mathcal{M}_{i}^{H}(j \omega)
\end{array}\right] .
$$

It is straightforward to verify that $\mathcal{W}^{H}(j \omega) \mathcal{W}(j \omega)=\mathcal{I}$, and hence

$$
J_{m}^{*}=\inf _{Q \in \mathcal{R} \mathcal{H}_{\infty}(\mathbb{D})}\left\|\mathcal{W}\left[I-\frac{1}{T} \mathcal{P}^{(m)} \mathcal{H} Q \mathcal{F}^{T}\right] \mathcal{R}\right\|_{\mathcal{L}_{2}\left(\Omega_{N}\right)}^{2} .
$$

Using the identities

$$
\begin{aligned}
\mathcal{M}_{i}^{H}(j \omega) \mathcal{R}(j \omega) & =-\frac{\sqrt{T}}{1-e^{j \omega T}} \frac{\left(P^{(m)} H H\right)_{d}\left(e^{-j \omega T}\right)}{\Theta_{d}\left(e^{-j \omega T}\right)} \\
\mathcal{F}^{T}(j \omega) \mathcal{R}(j \omega) & =\frac{T}{1-e^{-j \omega T}}(F H)_{d}\left(e^{j \omega T}\right) \\
\mathcal{R}^{H}(j \omega) \mathcal{R}(j \omega) & =\frac{T^{2}}{\left(1-e^{j \omega T}\right)\left(1-e^{-j \omega T}\right)}
\end{aligned}
$$

it follows from a standard, albeit tedious calculation of (A4) that:

$$
J_{m}^{*}=J_{m 1}^{*}+J_{s 1}
$$

with

$$
\begin{aligned}
J_{s 1} & :=\frac{T}{2 \pi} \int_{0}^{\omega_{s}} \frac{\left|\frac{\left(P^{(m)} H H\right)_{d}\left(e^{j \omega T}\right)}{\Theta_{d}\left(e^{j \omega T}\right)}\right|^{2}}{\left|1-e^{j \omega T}\right|^{2}} d \omega \\
J_{m 1}^{*} & :=\inf _{Q \in \mathcal{R H}_{\infty}(\mathbb{I D})}\left\|\frac{\frac{\left(P^{(m)} H H\right)_{d}^{\sim}}{\Theta_{d}^{\tilde{D}}}-z(F H)_{d} \Theta_{d} Q}{z-1}\right\|_{2}^{2}
\end{aligned}
$$

where $\left(P^{(m)} H H\right)_{d}(z):=\left(P^{(m)} H H\right)_{d}\left(z^{-1}\right), \Theta_{\tilde{d}}^{\sim}(z):=$ $\Theta_{d}\left(z^{-1}\right)$. As such, we compute $J_{m 1}^{*}$. Toward this end, we factorize $z\left(P^{(m)} H H\right)_{d}(z)$ and $(F H)_{d}(z)$, respectively, as

$$
\begin{aligned}
z\left(P^{(m)} H H\right)_{d}(z) & =L_{s}(z)\left(P^{(m)} H H\right)_{d}^{(m)}(z) \\
(F H)_{d}(z) & =L_{f}(z)(F H)_{d}^{(m)}(z),
\end{aligned}
$$

where $\left(P^{(m)} H H\right)_{d}^{(m)}(z)$ and $(F H)_{d}^{(m)}(z)$ are the minimum phase parts of $z\left(P^{(m)} H H\right)_{d}(z)$ and $(F H)_{d}(z)$, respectively, and

$$
\begin{aligned}
& L_{s}(z)=\prod_{i=1}^{m_{p}}\left(\frac{1-\bar{\sigma}_{i}}{1-\sigma_{i}}\right)\left(\frac{z-\sigma_{i}}{1-\bar{\sigma}_{i} z}\right) \\
& L_{f}(z)=\prod_{i=1}^{m_{f}}\left(\frac{1-\bar{\lambda}_{i}}{1-\lambda_{i}}\right)\left(\frac{z-\lambda_{i}}{1-\bar{\lambda}_{i} z}\right)
\end{aligned}
$$

are the all-pass factors. Write $\left[\left(P^{(m)} H H\right)_{d}^{(m)}\right] \sim(z):=$ $\left(P^{(m)} H H\right)_{d}^{(m)}\left(z^{-1}\right)$ and $L_{s}^{\sim}(z):=L_{s}\left(z^{-1}\right)$. Note that $L_{s}(1)=L_{f}(1)=1$. Note also that

$$
\left(P^{(m)} H H\right)_{d}(z)=\left(1-z^{-1}\right)\left(P^{(m)} R H\right)_{d}(z) .
$$

Hence, the transfer functions $\left(P^{(m)} H H\right)_{d}(z)$ and $\left(P^{(m)} R H\right)_{d}(z)$ share the same non-minimum phase zeros in $\mathbb{D}^{c}$. Furthermore, since $\left(P^{(m)} R H\right)_{d}(z)$ is a rational function, $\left(P^{(m)} H H\right)_{d}(z) \in \mathcal{R} \mathcal{H}_{\infty}(\mathbb{D})$. We may then write

$J_{m 1}^{*}=\inf _{Q \in \mathcal{R} \mathcal{H}_{\infty}(\mathbb{I})}\left\|\frac{\frac{L_{s}^{\sim}\left[\left(P^{(m)} H H\right)_{d}^{(m)}\right]^{\sim}}{\Theta_{\tilde{d}}}-L_{f}(F H)_{d}^{(m)} \Theta_{d} Q}{z-1}\right\|_{2}^{2}$

or alternatively

$$
J_{m 1}^{*}=\inf _{Q \in \mathcal{R} \mathcal{H}_{\infty}(\mathbb{D})}\left\|\frac{\frac{\left[\left(P^{(m)} H H\right)_{d}^{(m)}\right]^{\sim}}{\Theta_{\tilde{d}}^{\tilde{N}}}-L_{d}(F H)_{d}^{(m)} \Theta_{d} Q}{z-1}\right\|_{2}^{2}
$$

where $L_{d}(z):=L_{s}(z) L_{f}(z)$. Since, according to (A2), (A3)

$$
\frac{\frac{\left(P^{(m)} H H\right)_{d}^{(m)}\left(z^{-1}\right)}{\Theta_{d}\left(z^{-1}\right)}-\sqrt{T}}{z-1} \in \mathcal{H}_{2}^{\perp}(\mathbb{D})
$$

we obtain

$$
\begin{aligned}
& J_{m 1}^{*}=\left\|\frac{\frac{\left(P^{(m)} H H\right)_{d}^{(m)}\left(z^{-1}\right)}{\Theta_{d}\left(z^{-1}\right)}-\sqrt{T}}{z-1}\right\|_{2}^{2} \\
&+\inf _{Q \in \mathcal{R} \mathcal{H}_{\infty}(\mathbb{D})}\left\|\frac{\sqrt{T}-L_{d}(F H)_{d}^{(m)} \Theta_{d} Q}{z-1}\right\|_{2}^{2}
\end{aligned}
$$


Furthermore, following the development in [33], we note that

$$
\begin{aligned}
\left\|\frac{\sqrt{T}-L_{d}(F H)_{d}^{(m)} \Theta_{d} Q}{z-1}\right\|_{2}^{2}= & T\left\|\frac{L_{d}^{-1}-1}{z-1}\right\|_{2}^{2} \\
& +T\left\|\frac{1-\frac{1}{\sqrt{T}}(F H)_{d}^{(m)} \Theta_{d} Q}{z-1}\right\|_{2}^{2}
\end{aligned}
$$

and

$$
\left\|\frac{L_{d}^{-1}(z)-1}{z-1}\right\|_{2}^{2}=\sum_{i=1}^{m_{p}} \frac{\sigma_{i}+1}{\sigma_{i}-1}+\sum_{i=1}^{m_{f}} \frac{\lambda_{i}+1}{\lambda_{i}-1} .
$$

Denote

$$
\begin{aligned}
J_{s 2} & :=\left\|\frac{\frac{\left(P^{(m)} H H\right)_{d}^{(m)}(z)}{\Theta_{d}(z)}-\sqrt{T}}{z-1}\right\|_{2}^{2} \\
& =\frac{T}{2 \pi} \int_{0}^{\omega_{s}}\left|\frac{\frac{\left(P^{(m)} H H\right)_{d}^{(m)}\left(e^{j \omega T}\right)}{\Theta_{d}\left(e^{j \omega T}\right)}-\sqrt{T}}{e^{j \omega T}-1}\right|^{2} d \omega .
\end{aligned}
$$

It is then clear that

$J_{s d}^{*}=\tau+\sum_{i=1}^{m_{c}} \frac{2}{z_{i}}+T \sum_{i=1}^{m_{p}} \frac{\sigma_{i}+1}{\sigma_{i}-1}+T \sum_{i=1}^{m_{f}} \frac{\lambda_{i}+1}{\lambda_{i}-1}+J_{s 1}+J_{s 2}$.

It is also clear that the optimal tracking performance $J_{s d}^{*}$ can be achieved by the solution of the discrete-time model-matching problem

$$
\inf _{Q \in \mathcal{R} \mathcal{H}_{\infty}(\mathbb{D})}\left\|\frac{1-\frac{1}{\sqrt{T}}(F H)_{d}^{(m)}(z) \Theta_{d}(z) Q(z)}{z-1}\right\|_{2}^{2}
$$

which can be solved using standard $\mathcal{H}_{2}$ optimal control procedures (see, e.g., [13]), or based on a cheap control approach [29].

The remaining part of the proof proceeds by evaluating $J_{s 1}+$ $J_{s 2}$, which, by a direct calculation, is found to be

$$
J_{s 1}+J_{s 2}=\frac{T^{2}}{2 \pi} \int_{-\omega_{N}}^{\omega_{N}} \frac{1-\frac{1}{\sqrt{T}} \operatorname{Re}\left\{\frac{\left(P^{(m)} H H\right)_{d}^{(m)}\left(e^{j \omega T}\right)}{\Theta_{d}\left(e^{j \omega T}\right)}\right\}}{1-\cos \omega T} d \omega .
$$

Making the variable substitution $y=\tan (\omega T / 2)$, we have

$$
J_{s 1}+J_{s 2}=\frac{T}{2 \pi} \int_{-\infty}^{\infty} \frac{1-\frac{1}{\sqrt{T}} \operatorname{Re}\left\{\frac{\left(P^{(m)} H H\right)_{d}^{(m)}\left(\frac{1+j y}{1-j y}\right)}{\Theta_{d}\left(\frac{1+j y y}{1-j y}\right)}\right\}}{y^{2}} d y .
$$

Introduce the function

$$
f(s):=\frac{1}{\sqrt{T}} \cdot \frac{\left(P^{(m)} H H\right)_{d}^{(m)}\left(\frac{1+s}{1-s}\right)}{\Theta_{d}\left(\frac{1+s}{1-s}\right)} .
$$

Since $\left(P^{(m)} H H\right)_{d}^{(m)}(z) / \Theta_{d}(z)$ is analytic in $\mathbb{D}^{c}, f(s)$ is analytic in $\mathbb{C}_{+}$. Denote $f(j y)=u(y)+j v(y)$. Then the Hilbert transform [23]

$$
v\left(y_{0}\right)=\frac{y_{0}}{\pi} \int_{-\infty}^{\infty} \frac{u(y)-u\left(y_{0}\right)}{y^{2}-y_{0}^{2}} d y
$$

holds for any real number $y_{0}$. Note from (A2), (A3) that $f(0)=$ $u(0)=1$. Hence by invoking the Hilbert transform, we obtain

$$
\begin{aligned}
J_{s 1}+J_{s 2} & =\int_{-\infty}^{\infty} \frac{1-\frac{1}{\sqrt{T}} \operatorname{Re}\left\{\frac{\left(P^{(m)} H H\right)_{d}^{(m)}\left(\frac{1+j y}{1-j y}\right)}{\Theta_{d}\left(\frac{1+j y}{1-j y}\right)}\right\}}{y^{2}} d y \\
& =-\int_{-\infty}^{\infty} \frac{u(y)-u(0)}{y^{2}} d y \\
& =-\pi \lim _{y \rightarrow 0} \frac{v(y)}{y} .
\end{aligned}
$$

Based on this recognition, we then find

$$
\begin{aligned}
J_{s 1}+J_{s 2} & \left.=-\frac{T}{2} \lim _{y \rightarrow 0} \frac{\operatorname{Im}\left\{\frac{\left(P^{(m)} H H\right)_{d}^{(m)}\left(\frac{1+j y}{1-j y}\right)}{\theta_{d}\left(\frac{1+j y}{1-j y}\right)}\right\} \frac{1}{\sqrt{T}}}{y}\right)\left.\right|_{z=1} \\
& =-\frac{\sqrt{T}}{2} \operatorname{Im}\left\{\frac{d}{d z}\left(\frac{\left(P^{(m)} H H\right)_{d}^{(m)}(z)}{\theta_{d}(z)}\right)\right. \\
& =T\left[\left.\frac{d}{d y}\left(\frac{1+j y}{1-j y}\right)\right|_{y=0} ^{\prime}(1)\right. \\
\Theta_{d}(1) & \left.-\frac{\left\{\left(P^{(m)} H H\right)_{d}^{(m)}\right\}^{\prime}(1)}{\left(P^{(m)} H H\right)_{d}^{(m)}(1)}\right] .
\end{aligned}
$$

Note that $J_{h}(T)=J_{s 1}+J_{s 2}$, and hence the singular integral in $J_{h}(T)$ is bound to converge. To complete the proof we next resort to the Schwarz integral formula [23], which, when applied to the function $\Theta_{d}(z) / \Theta_{d}(1)$, yields

$$
\log \frac{\Theta_{d}(z)}{\Theta_{d}(1)}=\frac{1}{4 \pi} \int_{0}^{2 \pi} \frac{e^{j t}+z}{e^{j t}-z} \log \left|\frac{\Theta_{d}\left(e^{j t}\right)}{\Theta_{d}(1)}\right|^{2} d t
$$

By evaluating the derivative of $\log \left(\Theta_{d}(z) / \Theta_{d}(1)\right)$ at $z=1$, we obtain

$$
\begin{aligned}
\frac{\Theta_{d}^{\prime}(1)}{\Theta_{d}(1)} & =-\frac{1}{4 \pi} \int_{0}^{2 \pi} \frac{2}{\left(1-e^{j t}\right)\left(1-e^{-j t}\right)} \log \left|\frac{\Theta_{d}\left(e^{j t}\right)}{\Theta_{d}(1)}\right|^{2} d t \\
& =\frac{1}{2 \pi} \int_{0}^{\pi} \frac{\log \left|\frac{\Theta_{d}\left(e^{j t}\right)}{\Theta_{d}(1)}\right|^{2}}{1-\cos t} d t .
\end{aligned}
$$


Similarly

$$
\frac{\left\{\left(P^{(m)} H H\right)_{d}^{(m)}\right\}^{\prime}(1)}{\left(P^{(m)} H H\right)_{d}^{(m)}(1)}=\frac{1}{2 \pi} \int_{0}^{\pi} \frac{\left.|\log | \frac{\left(P^{(m)} H H\right)_{d}^{(m)}\left(e^{j t}\right)}{\left(P^{(m)} H H\right)_{d}^{(m)}(1)}\right|^{2}}{1-\cos t} d t .
$$

Since

$$
\begin{array}{r}
\left|\Theta_{d}\left(e^{j \omega T}\right)\right|^{2}=\frac{1}{T} \sum_{k=-\infty}^{\infty}\left|P_{k}^{(m)}(j \omega) H_{k}(j \omega)\right|^{2} \\
\left|\left(P^{(m)} H H\right)_{d}^{(m)}\left(e^{j \omega T}\right)\right|^{2}=\frac{1}{T^{2}}\left|\sum_{k=-\infty}^{\infty} P_{k}^{(m)}(j \omega) H_{k}^{2}(j \omega)\right|^{2}
\end{array}
$$

it follows from (A5) and (A6) that

$$
\begin{aligned}
& \frac{\Theta_{d}^{\prime}(1)}{\Theta_{d}(1)}-\frac{\left\{\left(P^{(m)} H H\right)_{d}^{(m)}\right\}^{\prime}(1)}{\left(P^{(m)} H H\right)_{d}^{(m)}(1)} \\
& =\frac{1}{2 \pi} \int_{0}^{\pi} \frac{1}{1-\cos t} \log \mid \frac{\Theta_{d}\left(e^{j t}\right)}{\left(P^{(m)} H H\right)_{d}^{(m)}\left(e^{j t}\right)} \\
& =\left.\left.\frac{T}{2 \pi} \int_{0}^{\omega_{N}} \frac{1}{1-\cos \omega T}\right|^{\frac{\left(P^{(m)} H H\right)_{d}^{(m)}(1)}{\Theta_{d}(1)}}\right|^{2} d t \\
& \quad \times \log \left\{T^{2} \frac{\sum_{k=-\infty}^{\infty}\left|P_{k}^{(m)}(j \omega) H_{k}(j \omega)\right|^{2}}{\left|\sum_{k=-\infty}^{\infty} P_{k}^{(m)}(j \omega) H_{k}^{2}(j \omega)\right|^{2}}\right\} d \omega .
\end{aligned}
$$

The proof is then completed by substituting

$$
H_{k}(j \omega)=\frac{1-e^{j \omega T}}{j\left(\omega+k \omega_{s}\right)} .
$$

\section{APPENDIX B}

PROOF OF THEOREM 2

Proof: In light of Theorem 1, it suffices to consider a delayfree plant and assume that $P(s)$ is minimum phase, and show that the corresponding tracking performance approaches zero at a linear rate, i.e.,

$$
J_{s d}^{*}=O(T)
$$

We accomplish this goal using a time-domain lifting approach [4], [30], [31], [36]. In particular, we rely on a formalism of [13], which studied a more specialized tracking problem under an $\mathcal{H}_{2}$ criterion.

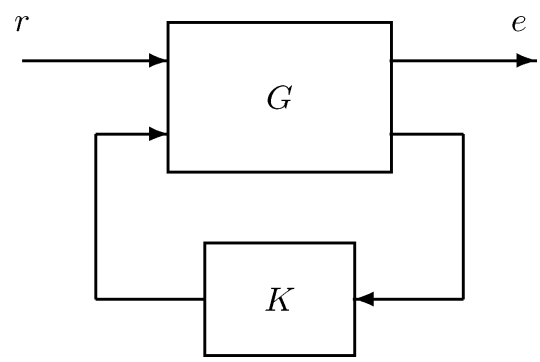

Fig. 2. Generalized feedback system.

Let us first consider a generalized continuous-time plant $G$, with the realization

$$
G=\left[\begin{array}{ccc}
A & B_{1} & B_{2} \\
C_{1} & 0 & D_{12} \\
C_{2} & 0 & 0
\end{array}\right]
$$

A generalized feedback system is shown in Fig. 2. With a continuous-time plant $G$ and a sampled-data controller $K$, the system can be converted into one with a discrete-time controller $K_{d}$ together with a generalized discrete-time plant $G_{d}$, which take the places of $G$ and $K$, respectively. The state-space realization of the discrete-time plant is given by

$$
G_{d}=\left[\begin{array}{ll}
G_{d_{11}} & G_{d_{12}} \\
G_{d_{21}} & G_{d_{22}}
\end{array}\right]=\left[\begin{array}{ccc}
A_{d} & B_{1} & B_{2 d} \\
C_{1 d} & 0 & D_{12 d} \\
C_{2} & 0 & 0
\end{array}\right]
$$

with

$$
A_{d}:=e^{A T}, \quad B_{2 d}:=\int_{0}^{T} e^{A t} B_{2} d t
$$

and

$$
\begin{aligned}
{\left[\begin{array}{ll}
C_{1 d} & D_{12 d}
\end{array}\right]^{T}\left[\begin{array}{ll}
C_{1 d} & D_{12 d}
\end{array}\right] } & \\
& :=\int_{0}^{T} e^{\underline{A}^{T} t}\left[\begin{array}{ll}
C_{1} & D_{12}
\end{array}\right]^{T}\left[\begin{array}{ll}
C_{1} & D_{12}
\end{array}\right] e^{\underline{A} t} d t
\end{aligned}
$$

where

$$
\underline{A}:=\left[\begin{array}{cc}
A & B_{2} \\
0 & 0
\end{array}\right] .
$$

To pose the tracking problem in the above generalized framework, we first construct

$$
G=\left[\begin{array}{cc}
1 / s & -P(s) \\
F(s) / s & -P(s) F(s)
\end{array}\right] .
$$

Let $\left(A_{p}, B_{p}, C_{p}, D_{p}\right)$ and $\left(A_{f}, B_{f}, C_{f}, D_{f}\right)$ be the minimal realizations of $P(s)$ and $F(s)$, respectively. In the remainder of 
this appendix, we impose Assumption 1, and assume additionally that $P(s)$ is minimum phase. Hence, $D_{f}=0$, since $F(s)$ is strictly proper. A minimal realization for $G(s)$ is given by

$$
\begin{aligned}
& A=\left[\begin{array}{ccc}
0 & 0 & 0 \\
0 & A_{p} & 0 \\
B_{f} & -B_{f} C_{p} & A_{f}
\end{array}\right] \\
& B=\left[\begin{array}{ll}
B_{1} & B_{2}
\end{array}\right]=\left[\begin{array}{cc}
1 & 0 \\
0 & B_{p} \\
0 & B_{f} D_{p}
\end{array}\right] \\
& C=\left[\begin{array}{l}
C_{1} \\
C_{2}
\end{array}\right]=\left[\begin{array}{ccc}
1 & -C_{p} & 0 \\
0 & 0 & C_{f}
\end{array}\right] \\
& D=\left[\begin{array}{cc}
0 & -D_{p} \\
0 & 0
\end{array}\right] .
\end{aligned}
$$

Discretize $G(s)$ to obtain $G_{d}(z)$, as outlined above. We note that ( $\left.A, B_{2}\right)$ is not stabilizable. However, there exist $K_{d}$ that internally stabilize $P(s) F(s)$. The set of all stabilizing controllers is given by

$$
\mathcal{K}_{d}=\left\{Q\left(1+G_{d_{22}} Q\right)^{-1}: Q \in \mathcal{R H}_{\infty}(\mathbb{D})\right\}
$$

The minimal tracking performance is then found to be

$$
J_{s d}^{*}=\inf _{Q \in \mathcal{R} \mathcal{H}_{\infty}(\mathbb{D})}\left\|G_{d_{11}}+G_{d_{12}} Q G_{d_{21}}\right\|_{2}^{2}
$$

We refer the above discretization scheme to [13] (Chap. 12), which was carried out therein for a similar tracking problem.

We next perform an asymptotic analysis ${ }^{3}$ on $J_{s d}^{*}$. Using the asymptotic expansion

$$
e^{\underline{A} t}=I+\underline{A} t+O\left(t^{2}\right)
$$

we find that for a sufficiently small $T>0$

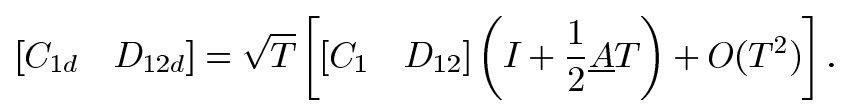

We may now calculate the transfer functions $G_{d_{11}}, G_{d_{21}}$, and $G_{d_{12}}$. It is immediate to find from the realization (B3) that

$$
\begin{aligned}
G_{d_{11}}(z) & =C_{1 d}\left(z I-A_{d}\right)^{-1} B_{1} \\
& =\sqrt{T}\left[\frac{1}{z-1}+O\left(T^{2}\right)\right] \\
G_{d_{21}}(z) & =C_{2}\left(z I-A_{d}\right)^{-1} B_{1} \\
& =C_{f}\left(z I-A_{f d}\right)^{-1} B_{f d} \frac{1}{z-1} \\
& =(F H)_{d}(z) \frac{1}{z-1} \\
G_{d_{12}}(z) & =C_{1 d}\left(z I-A_{d}\right)^{-1} B_{2 d}+D_{12 d} \\
& =-\sqrt{T}\left[C_{p}\left(z I-A_{p d}\right)^{-1} B_{p d}+D_{p}+O\left(T^{2}\right)\right] \\
& =-\sqrt{T}\left[(P H)_{d}(z)+O\left(T^{2}\right)\right] .
\end{aligned}
$$

${ }^{3}$ This asymptotic analysis was performed in [32], which unfortunately contains an error and hence led to an erroneous result.
Here we have taken $A_{p d}=e^{A_{p} T}, A_{f d}=e^{A_{f} T}$

$$
B_{p d}=\int_{0}^{T} e^{A_{p} t} B_{p} d t, \quad B_{f d}=\int_{0}^{T} e^{A_{f} t} B_{f} d t
$$

and the fact that $B_{p d}=O(T), B_{f d}=O(T)$. Thus, we find that

$$
\begin{aligned}
& \left|G_{d_{11}}+G_{d_{12}} Q G_{d_{21}}\right|^{2} \\
& \quad=T\left|\frac{1-\left[(P H)_{d}+O\left(T^{2}\right)\right](F H)_{d} Q}{z-1}+O\left(T^{2}\right)\right|^{2}
\end{aligned}
$$

Select $Q^{*} \in \mathcal{R H}_{\infty}(\mathbb{D})$ so that

$$
\begin{aligned}
\| \frac{1-(P H)_{d}(F H)_{d} Q^{*}}{z-1} & \|_{2}^{2} \\
& =\inf _{Q \in \mathcal{R H}_{\infty}(\mathbb{D})}\left\|\frac{1-(P H)_{d}(F H)_{d} Q}{z-1}\right\|_{2}^{2} \\
& =T \sum_{i=1}^{m_{d}} \frac{\sigma_{i}+1}{\sigma_{i}-1}
\end{aligned}
$$

where $\sigma_{i} \in \mathbb{D}^{c}$ are the non-minimum phase zeros of $(P H)_{d}(z)(F H)_{d}(z)$, which, according to [2], [3], [17], will approach to the zeros of the Euler polynomial. For this to be possible, however, it is necessary that $(P H)_{d}(F H)_{d} Q^{*}$ possesses an order $O(1)$ when $T \rightarrow 0$; in other words, the order of $Q^{*}$ cannot be lower than $O\left(1 / T^{2}\right)$. We are thus led to

$$
\begin{aligned}
J_{s d}^{*} & \leq T \inf _{Q \in \mathcal{R} \mathcal{H}_{\infty}(\mathbb{D})}\left\|\frac{1-(P H)_{d}(F H)_{d} Q}{z-1}\right\|_{2}^{2}+O\left(T^{2}\right) \\
& =T \sum_{i=1}^{m_{d}} \frac{\sigma_{i}+1}{\sigma_{i}-1}+O\left(T^{2}\right) .
\end{aligned}
$$

which, along with the recognition from Theorem 1 , that $J_{s d}^{*} \geq$ $O(T)$, establishes the claim $J_{s d}^{*}=O(T)$. This completes the proof.

\section{APPENDIX C}

PROOF OF THEOREM 3

Proof: We first note that for any $\theta \in[0, T), J(T-\theta)$ can be expressed as

$$
\begin{gathered}
J(T-\theta) \\
=\frac{1}{2 \pi} \int_{-\infty}^{\infty} \mid R(j \omega) e^{-j(T-\theta) \omega}-P^{\tau}(j \omega) H(j \omega) Q\left(e^{j \omega T}\right) \\
\quad \times\left.\frac{1}{T} \sum_{k=-\infty}^{\infty} F_{k}(j \omega) R_{k}(j \omega) e^{-j(T-\theta)\left(\omega+k \omega_{s}\right)}\right|^{2} d \omega \\
=\frac{1}{2 \pi} \int_{-\infty}^{\infty} \mid R(j \omega) e^{j \theta \omega}-e^{j \omega T} P^{\tau}(j \omega) H(j \omega) Q\left(e^{j \omega T}\right) \\
\times\left.\frac{1}{T} \sum_{k=-\infty}^{\infty} F_{k}^{\theta}(j \omega) R_{k}(j \omega)\right|^{2} d \omega
\end{gathered}
$$


where $F^{\theta}(s)=F(s) e^{-(T-\theta) s}$. It follows as in the proof of Theorem 1 that

$$
J(T-\theta)=\theta+\left\|\left[\mathcal{I}-\frac{1}{T} \mathcal{P}^{\tau} \mathcal{H} e^{j \omega T} Q\left(\mathcal{F}^{\theta}\right)^{T}\right] \mathcal{R}\right\|_{\mathcal{L}_{2}\left(\Omega_{N}\right)}^{2} .
$$

Note that for $\theta \in[0, T)$, a proper rational $\mathrm{ZOH}$-equivalent discretization exists for $F^{\theta}(s)[16$, p. 175] given as

$$
\left(F^{\theta} H\right)_{d}(z)=C_{f}\left(z I-A_{f d}\right)^{-1}\left(\Gamma_{2}+\frac{\Gamma_{1}}{z}\right)
$$

where

$$
\begin{aligned}
& \Gamma_{1}:=\int_{\theta}^{T} e^{A_{f} t} B_{f} d t=B_{f d}-B_{f}(\theta) \\
& \Gamma_{2}:=\int_{0}^{\theta} e^{A_{f} t} B_{f} d t=B_{f}(\theta) .
\end{aligned}
$$

In view of the convergence condition alluded to in Section II-B, we have

$$
\begin{aligned}
\frac{1}{T}\left(\mathcal{F}^{\theta}(j \omega)\right)^{T} \mathcal{R}(j \omega) & =\frac{1}{T} \sum_{k=-\infty}^{\infty} F_{k}^{\theta}(j \omega) R_{k}(j \omega) \\
& =\frac{\left(F^{\theta} H\right)_{d}\left(e^{j \omega T}\right)}{1-e^{-j \omega T}}
\end{aligned}
$$

Following the proof of Theorem 1, we are led to

$J(T-\theta)=\theta+\tau+\sum_{i=1}^{m_{c}} \frac{2}{z_{i}}+T \sum_{i=1}^{m_{p}} \frac{\sigma_{i}+1}{\sigma_{i}-1}+J_{h}(T)+J_{f}(\theta)$

where

$$
J_{f}(\theta):=\left\|\frac{\sqrt{T}-z \Theta_{d}(z) Q(z)\left(F^{\theta} H\right)_{d}(z)}{z-1}\right\|_{2}^{2}
$$

Since

$$
\int_{0}^{T} J(\theta) d \theta=\int_{0}^{T} J(T-\theta) d \theta
$$

we have

$$
J^{a}=\frac{1}{T} \int_{0}^{T} J(T-\theta) d \theta=\frac{T}{2}+J_{p}+\frac{1}{T} \int_{0}^{T} J_{f}(\theta) d \theta .
$$

Write $\hat{Q}(z)=\Theta_{d}(z) Q(z)$, and note that

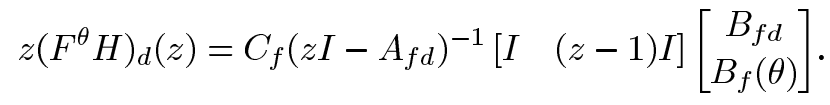

\section{Denote}

$$
\begin{aligned}
M_{f}(z) & :=C_{f}\left(z I-A_{f d}\right)^{-1}\left[\begin{array}{ll}
I & (z-1) I
\end{array}\right] \\
N(\theta) & :=\left[\begin{array}{c}
B_{f d} \\
B_{f}(\theta)
\end{array}\right] \\
\bar{N} & :=\frac{1}{T} \int_{0}^{T} N(\theta) d \theta \\
\Delta & :=\frac{1}{T} \int_{0}^{T} N(\theta) N^{T}(\theta) d \theta-\bar{N} \bar{N}^{T} .
\end{aligned}
$$

Then, a direct calculation yields the expression

$$
\begin{aligned}
\frac{1}{T} \int_{0}^{T}\left\|\sqrt{T}-\hat{Q}(z) M_{f}(z) N(\theta)\right\|_{2}^{2} d \theta \\
=\frac{T}{2 \pi} \int_{0}^{\omega_{s}}\left[T-\sqrt{T} \hat{Q}\left(e^{j \omega T}\right) M_{f}\left(e^{j \omega T}\right) \bar{N}-\sqrt{T} \bar{N} \hat{Q}\right. \\
\times\left(e^{-j \omega T}\right) M_{f}^{T}\left(e^{-j \omega T}\right)+\hat{Q}\left(e^{j \omega T}\right) M_{f}\left(e^{j \omega T}\right) \\
\left.\times\left(\Delta+\bar{N} \bar{N}^{T}\right) \hat{Q}\left(e^{-j \omega T}\right) M_{f}^{T}\left(e^{-j \omega T}\right)\right] d \omega .
\end{aligned}
$$

Furthermore,

$$
\Delta=\left[\begin{array}{cc}
0 & 0 \\
0 & \Sigma_{f}-B_{f a} B_{f a}^{T}
\end{array}\right]=\left[\begin{array}{c}
0 \\
\Lambda_{f}
\end{array}\right]\left[\begin{array}{ll}
0 & \Lambda_{f}^{T}
\end{array}\right]
$$

Since

$$
M_{f}(z)\left[\begin{array}{c}
B_{f d} \\
B_{f a}
\end{array}\right]=F_{a 1}(z), \quad M_{f}(z)\left[\begin{array}{c}
0 \\
\Lambda_{f}
\end{array}\right]=F_{a 2}(z)
$$

we obtain

$$
\begin{aligned}
\frac{1}{T} \int_{0}^{T} J_{f}(\theta) d \theta & =\left\|\frac{\sqrt{T}-\hat{Q} F_{a 1}}{z-1}\right\|_{2}^{2}+\left\|\frac{\hat{Q} F_{a 2}}{z-1}\right\|_{2}^{2} \\
& =\left\|\frac{\left[\begin{array}{ll}
\sqrt{T} & 0
\end{array}\right]-\hat{Q}\left[\begin{array}{ll}
F_{a 1} & F_{a 2}
\end{array}\right]}{z-1}\right\|_{2}^{2}
\end{aligned}
$$

Consequently, we have

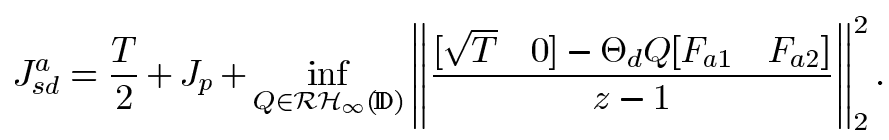

Conduct then the spectral factorization

$$
\Theta_{f}\left(e^{-j \omega T}\right) \Theta_{f}\left(e^{j \omega T}\right)=\left|F_{a 1}\left(e^{j \omega T}\right)\right|^{2}+\left\|F_{a 2}\left(e^{j \omega T}\right)\right\|_{2}^{2}
$$


and solve the $\mathcal{H}_{2}$ problem in the above equation, we obtain

$$
\begin{aligned}
J_{s d}^{a}=\frac{T}{2}+J_{p} & +\frac{T^{2}}{2 \pi} \int_{0}^{\omega_{s}} \frac{1-\left|\frac{F_{a 1}\left(e^{j \omega T}\right)}{\Theta_{f}\left(e^{j \omega T}\right)}\right|^{2}}{\left|e^{j \omega T}-1\right|^{2}} d \omega \\
& +\inf _{Q \in \mathcal{R H}_{\infty}(\mathbb{D})}\left\|\frac{\sqrt{T} \frac{F_{a 1}\left(z^{-1}\right)}{\Theta_{f}\left(z^{-1}\right)}-\Theta_{d}(z) Q(z)}{z-1}\right\|_{2}^{2} .
\end{aligned}
$$

Note that $\left|F_{a 1}(1) / \Theta_{f}(1)\right|=1$, and that with no loss of generality, we may take $F_{a 1}(1) / \Theta_{f}(1)=1$. Following the steps in evaluating $J_{m 1}^{*}$ (cf. Appendix A), we find that

$$
\begin{aligned}
\inf _{Q \in \mathcal{R} \mathcal{H}_{\infty}(\mathbb{D})}\left\|\frac{\sqrt{T} \frac{F_{a 1}\left(z^{-1}\right)}{\Theta_{f}\left(z^{-1}\right)}-\Theta_{d}(z) Q(z)}{z-1}\right\|_{2}^{2} \\
=\left\|\frac{\sqrt{T} \frac{F_{a 1}\left(z^{-1}\right)}{\Theta_{f}\left(z^{-1}\right)}-\sqrt{T} \frac{F_{a 1}(1)}{\Theta_{f}(1)}}{z-1}\right\|_{2}^{2} \\
=T \sum_{i=1}^{m_{a}} \frac{\gamma_{i}+1}{\gamma_{i}-1}+\left\|\frac{\sqrt{T} \frac{F_{a 1}^{(m)}(z)}{\Theta_{f}(z)}-\sqrt{T}}{z-1}\right\|_{2}^{2}
\end{aligned}
$$

where $F_{a 1}^{(m)}(z)$ is the minimum phase part of $F_{a 1}(z)$. In summary, we have shown that

$$
\begin{aligned}
J_{s d}^{a}= & \frac{T}{2}+J_{p}+T \sum_{i=1}^{m_{a}} \frac{\gamma_{i}+1}{\gamma_{i}-1}+T\left\|\frac{\frac{F_{a 1}^{(m)}(z)}{\Theta_{f}(z)}-1}{z-1}\right\|_{2}^{2} \\
& +\frac{T^{2}}{2 \pi} \int_{0}^{\omega_{s}} \frac{1-\left|\frac{F_{a 1}\left(e^{j \omega T}\right)}{\Theta_{f}\left(e^{j \omega T}\right)}\right|^{2}}{\left|e^{j \omega T}-1\right|^{2}} d \omega \\
= & \frac{T}{2}+J_{p}+T \sum_{i=1}^{m_{a}} \frac{\gamma_{i}+1}{\gamma_{i}-1} \\
& +\frac{T^{2}}{2 \pi} \int_{0}^{\omega_{s}} \frac{1-\operatorname{Re}\left\{\frac{F_{a 1}\left(e^{j \omega T}\right)}{\Theta_{f}\left(e^{j \omega T}\right)}\right\}}{1-\cos \omega T} d \omega .
\end{aligned}
$$

Mimicking the steps in calculating $J_{s 1}+J_{s 2}$, we obtain

$$
\begin{aligned}
\int_{0}^{\omega_{s}} \frac{1-\operatorname{Re}\left\{\frac{F_{a 1}\left(e^{j \omega T}\right)}{\Theta_{f}\left(e^{j \omega T}\right)}\right\}}{1-\cos \omega T} d \omega= & \int_{0}^{\omega_{N}} \frac{1}{1-\cos \omega T} \\
& \times \log \left(1+\frac{\left\|F_{a 2}\left(e^{j \omega T}\right)\right\|^{2}}{\left|F_{a 1}\left(e^{j \omega T}\right)\right|^{2}}\right) d \omega .
\end{aligned}
$$

The proof is thus completed.

\section{REFERENCES}

[1] M. Araki, Y. Ito, and T. Hagiwara, "Frequency response of sampleddata systems," Automatica, vol. 32, no. 4, pp. 483-497, Apr. 1996.
[2] K. J. Åström, P. Hagander, and J. Sternby, "Zeros of sampled systems," Automatica, vol. 20, no. 1, pp. 31-38, 1984.

[3] E. W. Bai and Y. Q. Wu, "Limiting zero distribution of sampled systems," Automatica, vol. 38, pp. 843-851, 2002.

[4] B. Bamieh and J. B. Pearson, "A general framework for linear periodic systems with application to $H_{\infty}$ sampled-data control," IEEE Trans. Automat. Control, vol. 37, no. 4, pp. 418-435, Apr. 1992.

[5] B. Bamieh and J. B. Pearson, "The $\mathcal{H}_{2}$ problem for sampled-data systems," Syst. Control Lett., vol. 19, pp. 1-12, 1992.

[6] M. J. Blachuta, "On zeros of pulse transfer functions," IEEE Tran. Automat. Control, vol. 44, no. 6, pp. 1229-1234, Jun. 1999.

[7] J. H. Braslavsky, G. Meinsma, R. H. Middleton, and J. S. Freudenberg, "On a key sampling formula relating the Laplace and $\mathcal{Z}$ transforms," Syst. Control. Lett., vol. 29, pp. 181-190, 1997.

[8] J. H. Braslavsky, R. H. Middleton, and J. S. Freudenberg, " $L_{2}$-induced norms and frequency gains of sampled-data sensitivity operators," IEEE Trans. Automat. Control, vol. 43, no. 2, pp. 252-258, Feb. 1998.

[9] J. Chen, S. Hara, and G. Chen, "Best tracking and regulation performance under control energy constraint," IEEE Trans. Automat. Control, vol. 48, no. 8, pp. 1320-1336, Aug. 2003.

[10] J. Chen, L. Qiu, and O. Toker, "Limitations on maximal tracking accuracy," IEEE Trans. Automat. Control, vol. 45, no. 2, pp. 326-331, Feb. 2000.

[11] J. Chen, L. Qiu, and O. Toker, "Limitations on maximal tracking accuracy, part 2: Tracking ramp and sinusoidal signals," in Proc. Amer. Control. Conf., Albuquerque, NM, Jun. 1997, pp. 1757-1761.

[12] T. Chen, "A simple derivation of the $\mathcal{H}_{2}$-optimal sampled-data controllers," Sys. Control Lett., vol. 20, pp. 49-56, 1993.

[13] T. Chen and B. A. Francis, Optimal Sampled-Data Control Systems. London, U.K.: Springer Verlag, 1995.

[14] T. Chen and B. A. Francis, " $\mathrm{H}_{2}$-optimal sampled-data control," IEEE Trans. Automat. Control, vol. 36, no. 4, pp. 387-397, Apr. 1991.

[15] T. Chen and B. A. Francis, "Linear time-varying $\mathcal{H}_{2}$-optimal control of sampled-data systems," Automatica, vol. 27, pp. 963-974, 1991.

[16] G. F. Franklin and J. D. Powell, Digital Control of Dynamic Systems. New York: Addison-Wesley, 1980.

[17] T. Hagiwara, "Analytic study on the intrinsic zeros of sampled-data systems," IEEE Trans. Automat. Control, vol. 41, no. 2, pp. 261-263, Feb. 1996.

[18] T. Hagiwara and M. Araki, "FR-operator approach to the $H_{2}$ analysis and synthesis of sampled-data systems," IEEE Trans. Automat. Control, vol. 40, no. 8, pp. 1411-1421, Aug. 1995.

[19] S. Hara, H. Fujioka, and P. T. Kabamba, "A hybrid state-space approach to aampled-data feedback control," Linear Algebra Appl., vol. 205-206, pp. 675-712, 1994.

[20] P. P. Khargonekar and N. Sivashankar, " $\mathcal{H}_{2}$ optimal control for sampled-data systems," Syst. Control Lett., vol. 17, pp. 425-436, 1991.

[21] H. Kwakernaak and R. Sivan, "The maximally achievable accuracy of linear optimal regulators and linear optimal filters," IEEE Trans. Automat. Control, vol. AC-17, no. 1, pp. 79-86, Feb. 1972.

[22] W. R. LePage, Complex Variables and the Laplace Transform for Engineers. New York: Dover, 1961.

[23] N. Levinson and R. M. Redheffer, Complex Variables. New York: McGraw-Hill, 1970.

[24] M. Morari and E. Zafiriou, Robust Process Control. Englewood Cliffs, NJ: Prentice Hall, 1989.

[25] K. Ogata, Discrete-Time Control Systems, 2nd ed. Englewood Cliffs, NJ: Prentice Hall, 1995.

[26] L. Qiu and E. J. Davison, "Performance limitations of nonminimum phase systems in the servomechanism problem," Automatica, vol. 29, pp. 337-349, 1993.

[27] L. Qiu and J. Chen, "Time domain performance limitations of feedback control," in Proc. 13th Int. Symp. Math. Theory Net. Syst., Padova, Italy, Jul. 1998, pp. 369-372.

[28] W. Su, L. Qiu, and J. Chen, "Fundamental performance limitations in tracking sinusoidal signals," IEEE Trans. Automat. Control, vol. 48, no. 8, pp. 1371-1380, Aug. 2003.

[29] W. Su, L. Qiu, and J. Chen, "Fundamental limit of discrete-time systems in tracking multi-tone sinusoidal signals," Automatica, vol. 43, no. 1, pp. 15-30, Jan. 2007.

[30] G. Tadmor, " $\mathcal{H}_{\infty}$ optimal sampled-data control in continuous-time systems," Int. J. Control, vol. 56, pp. 99-141, 1992. 
[31] H. T. Toivonen, "Sampled-data control of continuous-time systems with an $\mathcal{H}_{\infty}$ optimality criterion," Automatica, vol. 28, pp. 45-54, 1992.

[32] O. Toker and J. Chen, "Asymptotic tracking performance of sampleddata systems," in Proc. 2002 Amer. Control Conf., Anchorage, AK, May 2002, pp. 2967-2972.

[33] O. Toker, J. Chen, and L. Qiu, "Tracking performance limitations in LTI multivariable discrete-time systems," IEEE Trans. Circ. Syst. I, vol. 49, no. 5, pp. 657-670, May 2002.

[34] H. L. Trentelman and A. A. Stoorvogel, "Sampled-data and discrete-time $\mathcal{H}_{2}$ optimal control," SIAM J. Control Optim., vol. 33, pp. 834-862, 1995.

[35] M. Vidyasagar, Control System Synthesis: A Factorization Approach. Cambridge, MA: MIT Press, 1985.

[36] Y. Yamamoto, "A function space approach to sampled data control systems and tracking problems," IEEE Trans. Automat. Control, vol. 39, no. 4, pp. 703-713, Apr. 1994.

[37] Y. Yamamoto and P. P. Khargonekar, "Frequency response of sampled-data systems," IEEE Trans. Automat. Control, vol. 41, no. 2, pp. 166-176, Feb. 1996.

[38] C. Zhang and J. Zhang, " $\mathcal{H}_{2}$ performance of continuous time periodically time-varying controllers," Syst. Control Lett., vol. 32, pp. 209-221, 1997.

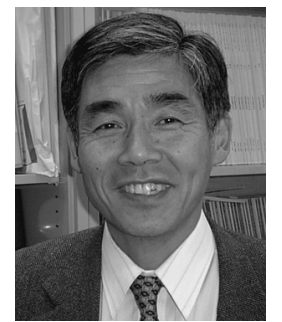

Shinji Hara (M'87-SM'04-F'06) was born in Izumo, Japan, in 1952. He received the B.S., M.S., and $\mathrm{Ph} . \mathrm{D}$. degrees in engineering from the Tokyo Institute of Technology, Tokyo, Japan, in 1974, 1976, and 1981, respectively.

In 1984, he joined the Tokyo Institute of Technology as an Associate Professor and has served as a Full Professor for ten years. Since 2002, he has been a Full Professor of the Department of Information Physics and Computing, The University of Tokyo. His current research interests are in robust control, sampled-data control, learning control, quantum control and computational aspects of control system design.

Dr. Hara received the George S. Axelby Outstanding Paper Award from the IEEE Control System Society in 2006, Best Paper Awards from The Society of Instrumentation and Control Engineers, Japan (SICE) several times. He was the General Chair of the CCA04 and Associate Editor of several international journals including IEEE TRANSACTIONS ON AUTOMATIC CONTROL AND Automatica. He is the Program Co-Chair of the 17th IFAC World Congress in Seoul, the Vice President of SICE, and a Fellow of SICE.

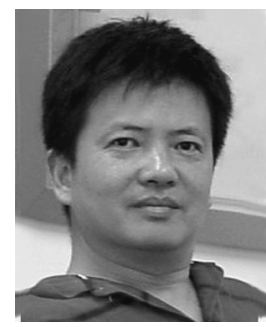

Li Qiu (F'07) received the B.Eng. degree from Hunan University, Hunan, China, in 1981, and the M.A.Sc. and Ph.D. degrees from the University of Toronto, Toronto, ON, Canada, in 1987 and 1990, respectively, all in electrical engineering.

He joined the Hong Kong University of Science and Technology, Hong Kong, China, in 1993, where he is now a Professor of electronic and computer engineering. His research interests include system, control, information theory, and mathematics for information technology.

Dr. Qiu served as an Associate Editor of the IEEE TRANSACTIONS ON Automatic Control and Automatica. He is now a Distinguished Lecturer of IEEE Control Systems Society and the General Chair of the 7th Asian Control Conference, which is to be held in Hong Kong in 2009.

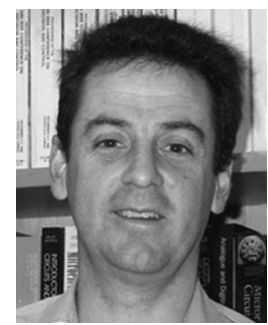

Richard H. Middleton (F'99) was born in Newcastle, Australia, on December 10, 1961. He received the B.Sc., B.Eng., and Ph.D. degrees from the University of Newcastle, Newcastle, Australia, in 1983 , 1984, and 1987, respectively

He served as an Associate Editor of Automatica. He was Head of the Electrical and Computer Engineering Department, University of Newcastle. He is currently a Research Professor at the Hamilton Institute, The National University of Ireland, Maynooth. His research interests include a range of control systems theory and applications.

Dr. Middleton has served as an Associate Editor for the IEEE TRANSACTIONS on Automatic CONTROL and the IEEE TRANSACTIONS ON CONTROL AND Systems TeChNOLOGY. He has been a Vice President and Distinguished Lecturer of the IEEE CSS Society. 